\title{
Intracardiac Echocardiography-Guided Cardiac Tumor Biopsy
}

\author{
Taiki Higo, MD; Masao Takemoto, MD; Kiyohiro Ogawa, MD; Shujiro Inoue, MD;
} Ken-ichi Eshima, MD; Hideo Tada, MD; Kenji Sunagawa, MD

\begin{abstract}
A 63-year-old woman was admitted to hospital with the chief complaint of new onset chest discomfort and pretibial pitting edema. Transthoracic echocardiography revealed a large invasive tumor on the heart protruding into the right atrium and right ventricle, which obstructed the outflow tract. She underwent transvenous $9 \mathrm{Fr}, 9-\mathrm{MHz}$ ultra intracardiac echocardiography (ICE) (EP Technologies, Boston Scientific Corporation, San Jose, CA, USA) guided biopsy, and a diagnosis of malignant lymphoma was established from the specimen obtained. ICE-guided cardiac tumor biopsy may be one of the most useful strategies for diagnosis of cardiac tumors. (Circ J 2009; 73:
\end{abstract} $381-383)$

Key Words: Biopsy; Cardiac tumor; Diagnosis; Intracardiac echocardiography; Malignant lymphoma

$\Delta$ though primary cardiac tumors are relatively rare, physicians unexpectedly encounter them in clinical practice and it is important to establish a histopathological diagnosis in order to treat them adequately; however, it is difficult to do so non-invasively. We describe a case of a primary cardiac malignant lymphoma that was easily and safely diagnosed histopathologically after a transvenous intracardiac echocardiography (ICE)-guided biopsy.

\section{Case Report}

A 63-year-old woman was admitted to hospital with the chief complaint of new onset chest discomfort and pretibial pitting edema. On physical examination, dilated jugular veins and pretibial pitting edema were noted. Auscultation revealed a cardiac ejection systolic murmur and normal vesicular lung sounds. Chest X-ray revealed cardiomegaly (cardiothoracic ratio $=64 \%$ ), and the electrocardiogram demonstrated right-axis deviation, right bundle blanch block, low voltage in the limb leads, and poor $\mathrm{R}$ wave progression in the precordial leads. Transthoracic echocardiography (TTE) revealed a large invasive cardiac tumor protruding into the right atrium (RA) and right ventricle (RV), obstructing the outflow tract (RVOT) (Figs 1A,B). There was also moderate pericardial effusion without any hemodynamic significance. Contrast computed tomography (CT) also showed the large cardiac tumor measuring $76 \times 37 \mathrm{~mm}$ in diameter and stretching from the RA to the $\mathrm{RV}$ and pulmonary artery (Fig 2). Pericardial effusion, as

(Received January 15, 2008; revised manuscript received March 5, 2008; accepted April 29, 2008; released online December 5, 2008)

Department of Cardiovascular Medicine, Kyushu University Hospital, Fukuoka, Japan

There is no conflict of interest related to this study.

Mailing address: Masao Takemoto, MD, Department of Cardiovascular Medicine, Kyushu University Hospital, 3-1-1 Maidashi, Higashiku, Fukuoka 812-8582, Japan. E-mail: matakemo@ cardiol.med. kyushu-u.ac.jp

All rights are reserved to the Japanese Circulation Society. For permissions, please e-mail: cj@j-circ.or.jp
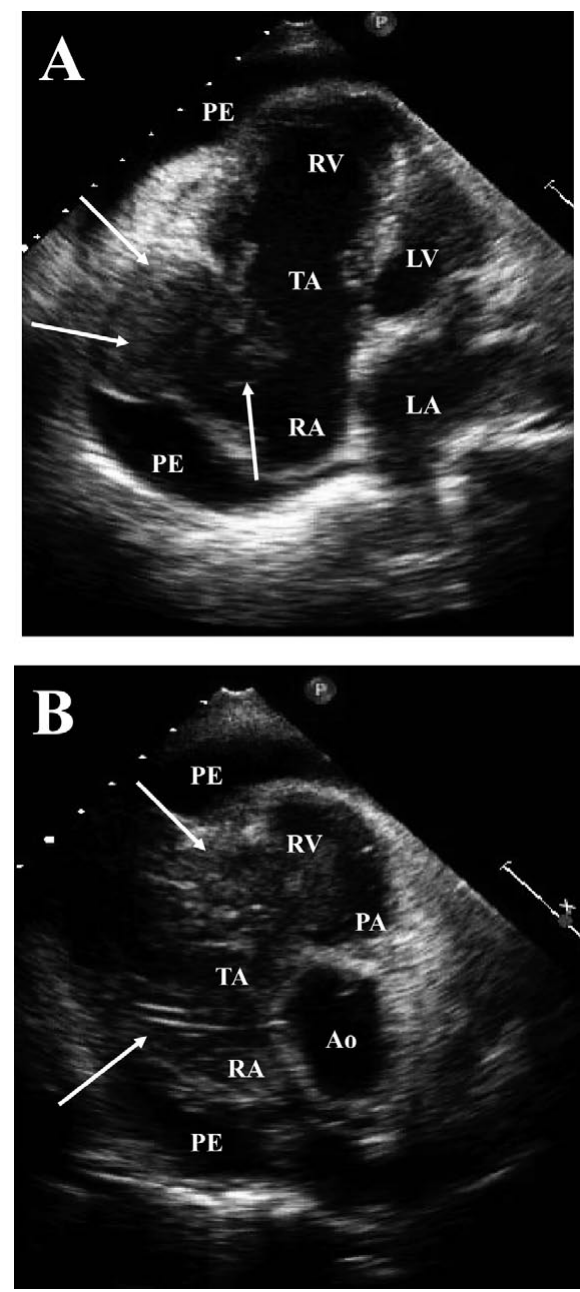

Fig 1. Two-dimensional transthoracic echocardiograms on admission in the apical 4-chamber view (A) and short-axis view (B) show the large invasive cardiac tumor (white arrows) protruding into the right atrium (RA) and right ventricle (RV), and causing an RV outflow tract obstruction. PA, pulmonary artery; PE, pericardial effusion; TA, tricuspid valve annulus; LA, left atrium; LV, left ventricle; Ao, aorta. 


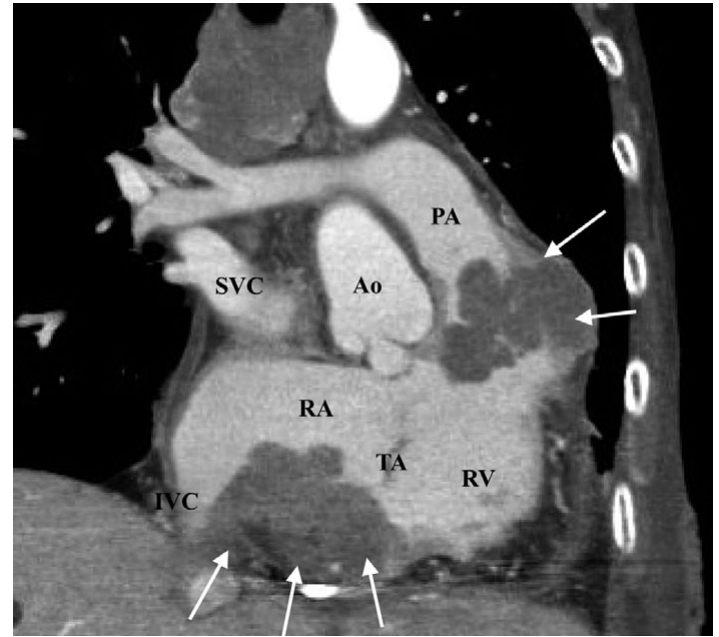

Fig 2. Contrast computed tomographic image shows the cardiac tumor (white arrows) protruding into the right atrium (RA) and right ventricle (RV), and causing an RV outflow tract obstruction. Ao, aorta; PA, pulmonary artery; TA, tricuspid valve annulus; SVC, superior vena cava; IVC, inferior vena cava.
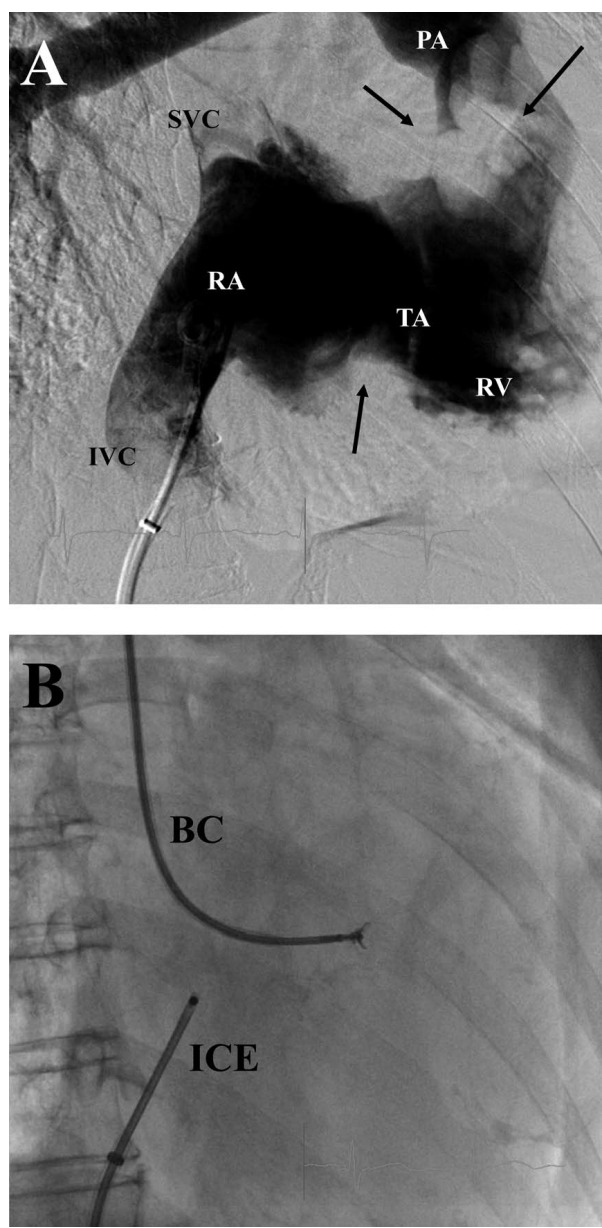

Fig 3. Right anterior oblique (RAO) view of a right atriogram (A) showing the cardiac tumor (black arrows) protruding into the right atrium (RA) and right ventricle (RV), and causing an RV outflow tract obstruction. RAO view of a fluoroscopic image (B) shows the location of the biopsy catheter (BC) next to the cardiac tumor and the intracardiac echocardiography (ICE) catheter in the RA. PA, pulmonary artery; TA, tricuspid valve annulus; SVC, superior vena cava; IVC, inferior vena cava.

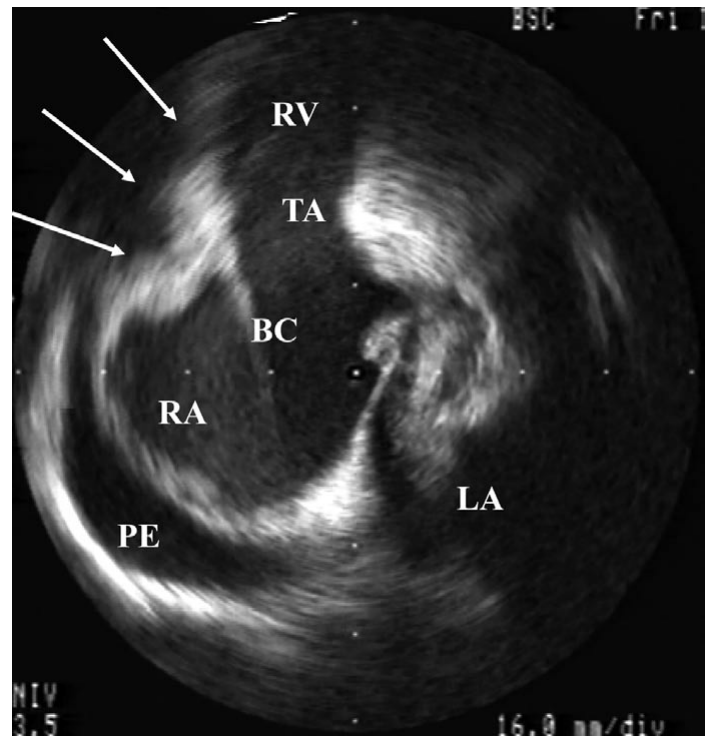

Fig 4. Intracardiac echocardiographic image shows the biopsy catheter $(\mathrm{BC})$ next to the cardiac tumor (white arrows) protruding into the right atrium (RA) and right ventricle (RV). PE, pericardial effusion; TA, tricuspid valve annulus; LA, left atrium.

well as mediastinal and mesenteric lymphadenopathy, were revealed. Because the cytology examination from the pericardial effusion was negative, a percutaneous cardiac tumor biopsy was performed in order to establish a histopathological diagnosis. Right atriography also revealed the cardiac tumor protruding into the RA and RV (Fig 3A). A 6Fr cardiac biopsy catheter (Technowood Biopsy Forceps, Tonokura Ika Kogyo Co Ltd, Tokyo, Japan) and 9Fr, 9-MHz Ultra ICE catheter (EP Technologies, Boston Scientific Corporation, San Jose, CA, USA) were percutaneously inserted into the RA via the right jugular vein and right femoral vein, respectively (Fig 3B). Because ICE images enabled detailed examination of the intracardiac structures, as well as an accurate surveillance of the positioning of the biopsy catheter (Fig 4), cardiac tumor specimens were easily and safely taken without any complications, pain, or exposure of the echocardiographer to radiation. Histopathological analysis showed diffuse large B-cell lymphoma. The patient underwent R-THPCOP chemotherapy (rituximab, pirarubicin, cyclosporin, vincristine, and prednisolone). During the first month following the treatment, her condition improved, which correlated with the finding of a decrease in the tumor size, and resolution of the pericardial effusion and RVOT obstruction on TTE. She has remained well under chemotherapy as an outpatient.

\section{Discussion}

Primary cardiac tumors are relatively rare with an incidence of $0.002-0.3 \%$ in autopsy series $;{ }^{1}$ however, physicians unexpectedly encounter them in clinical practice and although the exact incidence of each specific tumor type can not be stated, approximately $75 \%$ of all cardiac tumors are histologically benign and the remainder are malignant? Sarcomas are the most common malignant primary cardiac tumors and primary cardiac lymphomas are much less common, 3 with a relative incidence of only $2 \%$ of primary cardiac tumors.

The available and useful noninvasive diagnostic tools 
such as echocardiography, CT, and magnetic resonance imaging can provide much important information regarding the size, shape, composition, attachment, and surface characteristics of tumors, 4,5 which are important considerations for surgical excision, but surgery is not an effective treatment for the great majority of malignant cardiac tumors, because of the large mass of cardiac tissue involved, as in the present case, and because all cardiac tumors have the potential for causing life-threatening complications, including embolization. Thus, it is critically important to establish a histopathlogical diagnosis for instigation of appropriate treatment as soon as possible.

Previous reports demonstrate that a transvenous biopsy under TTE or transesophageal echocardiographic (TEE) guidance ${ }^{4,7,8}$ is a useful diagnostic approach. Although TTE is the most noninvasive and convenient technique, TEE can provide superior imaging to TTE, especially of posteriorly located structures? However, TEE sometimes causes the patient pain if performed without anesthesia, and for that reason it may usually requires local pharyngeal anesthesia and sometimes general sedation. Moreover, the operator of the TTE or TEE is at risk of exposure to radiation during the procedure.

ICE is a new technique based on the use of an ultrasonic diagnostic catheter that can be introduced through the femoral vein and provides a 2-dimensional view 10 Image acquisition is usually from within the RA or RV. Thus, ICE enables complete examination of the inter-atrial and -ventricular structures, as well as an accurate surveillance of the positioning of intracardiac devices ${ }^{11}$ such as a biopsy catheter. A major advantage of ICE is its potential to provide superior information to TEE ${ }^{10,11}$ as well as avoiding general sedation and exposure of the echocardiographer to radiation. Therefore, ICE may be one of the most useful diagnostic strategies and should be considered when trying to diagnose the histopathology of cardiac tumors. At present, however, the main limiting factor is its high cost, which is related to the single-use catheters.

\section{Acknowledgments}

We thank Kazutaka Yamaguchi for his technical assistance for intracardiac echocardiography, and John Martin for his linguistic assistance with this paper.

\section{References}

1. Reynen K. Frequency of primary tumors of the heart. Am J Cardiol 1996; 77: 107.

2. Lam KY, Dickens P, Chan AC. Tumors of the heart: A 20-year experience with a review of 12,485 consecutive autopsies. Arch Pathol Lab Med 1993; 117: 1027-1031.

3. Kasai K, Kuwao S, Sato Y, Murayama M, Harano Y, Kameya T. Case report of primary cardiac lymphoma: The applications of PCR to the diagnosis of primary cardiac lymphoma. Acta Pathol Jpn 1992; 42: 667-671.

4. Abramowitz Y, Hiller N, Perlman G, Admon D, Beeri R, ChajekShaul $\mathrm{T}$, et al. The diagnosis of primary cardiac lymphoma by right heart catheterization and biopsy using fluoroscopic and transthoracic echocardiographic guidance. Int J Cardiol 2007; 118: e39-e40.

5. Sato Y, Matsumoto N, Kinukawa N, Matsuo S, Komatsu S, Kunimasa $\mathrm{T}$, et al. Successful treatment of primary cardiac B-cell lymphoma: Depiction at multislice computed tomography and magnetic resonance imaging. Int J Cardiol 2006; 113: E26-E29.

6. Harvey WP. Clinical aspects of cardiac tumors. Am J Cardiol 1968; 21: $28-43$.

7. Kang SM, Rim SJ, Chang HJ, Choi D, Cho SY, Cho SH, et al. Primary cardiac lymphoma diagnosed by transvenous biopsy under transesophageal echocardiographic guidance and treated with systemic chemotherapy. Echocardiography 2003; 20: 101-103.

8. Jurkovich D, de Marchena E, Bilsker M, Fierro-Renoy C, Temple D, Garcia H. Primary cardiac lymphoma diagnosed by percutaneous intracardiac biopsy with combined fluoroscopic and transesophageal echocardiographic imaging. Catheter Cardiovasc Interv 2000; 50: 226-233.

9. Edwards LC 3rd, Louie EK. Transthoracic and transesophageal echocardiography for the evaluation of cardiac tumors, thrombi, and valvular vegetations. Am J Card Imaging 1994; 8: 45-58.

10. Girod G, Delabays A, Roguelov C, Renders F, Van de Walle S, Vogt $\mathrm{P}$, et al. Intracardiac echocardiography: A new tool for interventional cardiology. Rev Med Suisse 2007; 3: 1696-1701.

11. Brochet E, Habib G. Intracardiac echocardiography during percutaneous closure of atrial septal defect and patent foramen ovale. Arch Mal Coeur Vaiss 2005; 98: 25-28. 